\title{
Corrigendum: MONTI: A Multi-Omics Non-Negative Tensor Decomposition Framework for Gene-Level Integrative Analysis
}

\author{
Inuk Jung ${ }^{1 *}$, Minsu Kim ${ }^{2}$, Sungmin Rhee ${ }^{3}$, Sangsoo $\mathrm{Lim}^{4}$ and Sun $\mathrm{Kim}^{2,3,4 *}$ \\ ${ }^{1}$ Department of Computer Science and Engineering, Kyungpook National University, Daegu, South Korea, ${ }^{2}$ Computing and \\ Computational Sciences Directorate, Oak Ridge National Laboratory, Oak Ridge, TN, United States, ${ }^{3}$ Department of Computer \\ Science and Engineering, Seoul National University, Seoul, South Korea, ${ }^{4}$ Interdisciplinary Program in Bioinformatics, Seoul \\ National University, Seoul, South Korea
}

Keywords: feature selection, tensor decomposition, cancer, multi-omics, integrative analysis

\section{A corrigendum on}

\section{OPEN ACCESS}

Approved by: Frontiers Editorial Office, Frontiers Media SA, Switzerland

*Correspondence:

Inuk Jung

inukjung@knu.ac.kr Sun Kim

sunkim.bioinfo@snu.ac.kr

Specialty section:

This article was submitted to

Computational Genomics,

a section of the journal

Frontiers in Genetics

Received: 17 September 2021 Accepted: 30 September 2021

Published: 25 October 2021

Citation:

Jung I, Kim M, Rhee S, Lim S and Kim S (2021) Corrigendum: MONTI: A

Multi-Omics Non-Negative Tensor

Decomposition Framework for Gene-

Level Integrative Analysis.

Front. Genet. 12:778490.

doi: 10.3389/fgene.2021.778490
MONTI: A Multi-Omics Non-Negative Tensor Decomposition Framework for Gene-Level Integrative Analysis

by Jung, I., Kim, M., Rhee, S., Lim, S., and Kim, S. (2021). Front. Genet. 12:682841. doi: 10.3389/fgene. 2021.682841

There is an error in the Funding statement. The correct number for "the Basic Science Research Program through the National Research Foundation of Korea (NRF) funded by the Ministry of Education" is "2020M3C9A5085604." Corrected statement is given below:

This research was supported by the Bio \& Medical Technology Development Program of the National Research Foundation (NRF) funded by the Korean government (MSIT) (2019M3E5D3073365), the Collaborative Genome Program for Fostering New Post-Genome Industry of the National Research Foundation (NRF) funded by the Ministry of Science and ICT (MSIT) (No. NRF-2014M3C9A3063541), and the Basic Science Research Program through the National Research Foundation of Korea (NRF) funded by the Ministry of Education (2020M3C9A5085604).

The authors apologize for this error and state that this does not change the scientific conclusions of the article in any way. The original article has been updated.

Publisher's Note: All claims expressed in this article are solely those of the authors and do not necessarily represent those of their affiliated organizations, or those of the publisher, the editors and the reviewers. Any product that may be evaluated in this article, or claim that may be made by its manufacturer, is not guaranteed or endorsed by the publisher.

Copyright (c) 2021 Jung, Kim, Rhee, Lim and Kim. This is an open-access article distributed under the terms of the Creative Commons Attribution License (CC BY). The use, distribution or reproduction in other forums is permitted, provided the original author(s) and the copyright owner(s) are credited and that the original publication in this journal is cited, in accordance with accepted academic practice. No use, distribution or reproduction is permitted which does not comply with these terms. 